\title{
ANATOMIA DA MADEIRA DE DEZESSEIS ESPÉCIES DE EUPHORBIACEAE LATO SENSU, COM BASE NA ANÁLISE DE AGRUPAMENTO DE CARACTERES ANATÔMICOS ${ }^{1}$
}

\author{
ANELISE MARTA SIEGLOCH² JOSÉ NEWTON CARDOSO MARCHIORI ${ }^{3}$ \\ SIDINEI RODRIGUES DOS SANTOS ${ }^{4}$
}

\section{RESUMO}

No presente estudo são analisadas dezesseis espécies de Euphorbiaceae lato sensu, nativas ou cultivadas no Rio Grande do Sul. Para o reconhecimento de grupos distintos foi realizada uma análise de agrupamento com o auxilio do software R, com base em matriz de dados binários dos caracteres anatômicos. No dendrograma observam-se dois grupos distintos: o primeiro, formado por Hieronyma alchorneoides, e o segundo pelas demais espécies estudadas. As espécies mais similares foram: Euphorbia pulcherrima e Euphorbia tirucalli; Sapium glandulosum e Sapium haematospermum; Croton dracunculoides e Croton pycnocephalus.

Palavras-chave: Anatomia da Madeira, Análise de Agrupamento, Euphorbiaceae.

\begin{abstract}
[Wood anatomy of sixteen species of Euphorbiaceae sensu lato, based on cluster analysis of anatomical features].

Sixteen species of Euphorbiaceae sensu lato were analyzed, both native and cultivated in Rio Grande do Sul state (Brazil). To recognize distinct groups, a cluster analysis was performed with the help of software R, based on a binary data matrix of anatomical characters. Two distinct groups can be observed in dendrogram: the first one, with Hieronyma alchorneoides, and the second gathering all the other fifteen species. The most similar species were: Euphorbia pulcherrima and Euphorbia tirucalli; Sapium glandulosum and Sapium haematospermum; Croton dracunculoides and Croton pycnocephalus.
\end{abstract}

Key words: Euphorbiaceae, Cluster Analysis, Wood Anatomy.

\section{INTRODUÇÃO}

A família Euphorbiaceae, uma das maiores das Angiospermas Eudicotiledôneas, inclui ervas, arbustos, árvores e lianas, geralmente com látex branco e, por vezes, espinescentes e de aspecto áfilo, à semelhança de Cactáceas (Schultz, 1943; Souza \& Lorenzi, 2008; Judd et al., 2009).

1 Recebido para publicação em 14/01/2013 e aceito para publicação em 10/04/2013.

2 Mestranda do Curso de Pós-Graduação em Engenharia Florestal. Bolsista CAPES. Universidade Federal de Santa Maria, CEP 97105-900. Santa Maria, RS, Brasil. anesiegloch@yahoo.com.br

3 Engenheiro Florestal, Dr. Bolsista de Produtividade em Pesquisa (CNPq - Brasil). Professor Titular do Departamento de Ciências Florestais, Universidade Federal de Santa Maria. Santa Maria, RS, Brasil.

4 Biólogo, Dr. Núcleo de Estudos Botânicos Balduino Rambo, UFSM.
Reconhecida, tradicionalmente, como agrupamento heterogêneo e com relações filogenticas ainda não definitivamente compreendidas, o estudo da estrutura genética de diversos gêneros da família levou, recentemente, ao reconhecimento de outras três famílias na mesma: Phyllanthaceae, Picrodendraceae e Putranjivaceae (APG II 2003; Wurdack et al., 2005; APG III 2009, Judd et al., 2009), as quais coincidem, em boa parte, com as delimitações clássicas de algumas subfamílias, propostas por Webster (1994).

Em Euphorbiaceae permaneceram as espécies com um único óvulo por lóculo, sementes geralmente ariladas, flores unissexuais, estiletes mais ou menos divididos e frutos esquizocárpicos (JUDD et al., 2009). As Euphorbiaceae, por sua vez, compreendem três subfamílias: Acalyphoideae, com plantas carentes de látex; Crotonoideae, com látex branco ou colorido, não 
cáustico; e Euphorboideae, com látex branco, frequentemente cáustico (Wurdack et al., 2005; Judd et al., 2009).

As espécies biovuladas foram transferidas para as atuais famílias Phyllanthaceae (plantas sem carúncula nas sementes, desprovidas de látex e nectários extraflorais), Picrodendraceae (sementes geralmente com carúncula) e Putranjivaceae, com fruto do tipo drupa (Souza \& Lorenzi, 2008).

Com distribuição pantropical, as Euphorbiaceae stricto senso, compreendem 220 gêneros e cerca de 6.100 espécies (Judd et al., 2008). Trata-se de uma das famílias mais numerosas nas formações florestais brasileiras, com 70 gêneros e cerca de 1000 espécies (Souza \& Lorenzi, 2008). No gênero Croton, abundante em quase todos os ecossistemas, destacase Croton urucurana, espécie típica de florestas ciliares. Espécie polimórfica, Alchornea triplinervea apresenta ampla distribuição geográfica, ocorrendo em quase todas as formações naturais; outros gêneros bastante comuns, são: Sebastiania, Sapium e Actinostemon (Souza \& Lorenzi, 2008).

Com distribuição pantropical, as Phyllanthaceae reúnem cerca de 60 gêneros e 1800 espécies, sendo a segunda família mais numerosa após a divisão das Euphorbiaceae lato sensu, contando, no Brasil, com 13 gêneros e 100 espécies. Destas, salientam-se: Phyllanthus, o gênero do quebra-pedras, muito utilizado na medicina popular; e Hieronyma alchorneoides, a iricurana da porção sul da Mata Atlântica (Souza \& Lorenzi, 2008).

Com distribuição pantropical, as Picrodendraceae compreendem cerca de 30 gêneros e 100 espécies. No Brasil, encontram-se apenas dois gêneros e quatro espécies, salientando-se Piranhea e Podocalyx, com espécies na região amazônica (Souza \& Lorenzi, 2008).

De distribuição paleotropical, as Putranjivaceae incluem três gêneros e cerca de 200 espécies, das quais três espécies de Drypetes na Amazônia e na Mata Atlântica do Nordeste brasileiro (Souza \& Lorenzi, 2008).
$\mathrm{Na}$ flora arbórea sul-riograndense apenas as famílias Euphorbiaceae e Phyllanthaceae estão representadas. Segundo Sobral et al. (2006), a primeira conta com 11 gêneros (Alchornea, Croton, Gymnanthes, Manihot, Pachystroma, Pausandra, Pera, Sapium, Sebastiania, Stillingia, Tetrorchidium) e a segunda por Hieronyma, Margaritaria e Phyllanthus.

No presente estudo serão analisadas dezesseis espécies de Euphorbiaceae e Phyllanthaceae, nativas ou cultivadas no estado do Rio Grande do Sul, com objetivo de agrupar e elaborar uma chave dicotômica para as mesmas, com base em caracteres anatômicos do lenho.

\section{REVISÃO DE LITERATURA}

Com relação à anatomia da madeira, cabe ressaltar, de início, que Metcalfe \& Chalk (1972) distinguiram na antiga família Euphorbiaceae as subfamílias Crotonoideae e Phyllanthoideae, com dois grupos na última.

O grupo "A" das Phyllanthoideae, designado "Tipo Aparosa", foi identificado com base nos seguintes caracteres: elementos vasculares de comprimento médio a muito longos; placas de perfuração geralmente escalariforme; pontoações intervasculares pequenas e diminutas; pontoações raio-vasculares grandes e alongadas; parênquima abundante, difuso, com séries de 8-16 células; raios heterocelulares de dois tamanhos distintos, com até 4-17 células de largura, alguns milímetros de altura e células marginais, não raro com mais de 10 fileiras de células eretas; e fibras muito longas, de paredes espessas, com pontoações simples (Metcalfe \& Chalk, 1972).

O grupo "B" das Phyllanthoideae, designado "Tipo Glochidion", foi identificado pelos seguintes detalhes anatômicos: elementos vasculares de comprimento médio a moderadamente longos; placas de perfuração simples; pontoações intervasculares diminutas a grandes; pontoações raio-vasculares grandes e alongadas ou pequenas e arredondadas; parênquima tipicamente ausente, raramente paratraqueal; raios 
heterogêneos, por vezes de 2 tamanhos distintos, com até 11 células de largura, $2 \mathrm{~mm}$ de altura e 10 ou mais fileiras de células marginais eretas; e fibras septadas de paredes finas a moderadamente espessas, de comprimento médio a moderadamente longo (Metcalfe \& Chalk, 1972).

Para as Crotonoideae, subfamília que inclui os gêneros Bernardia, Croton, Euphorbia e Sapium, entre outros, Metcalfe \& Chalk (1972) referem os seguintes caracteres anatômicos: elementos vasculares de comprimento médio a moderadamente curto; placas de perfuração simples; pontoações intervasculares médias a grandes; pontoações raio-vasculares grandes e alongadas (frequentemente escalariformes) ou semelhantes às intervasculares; parênquima abundante, apotraqueal, com células esparsas ou faixas contínuas, com 1-2 células de largura; séries parenquimáticas de 8 células ( 4 , em alguns gêneros); raios marcadamente heterogêneos, com 2-3 (por vezes 4-5) células de largura ou exclusivamente unisseriados, com células marginais e, não raro, 10 ou mais fileiras de células eretas, por vezes com mais de uma parte multisseriada por raio; espaços intercelulares e tubos laticíferos, ocasionais; e fibras de paredes finas a moderadamente espessas, de comprimento médio a moderadamente curto, com pontoações simples ou pequenas aréolas.

Para os gêneros de Euphorbiaceae lato sensu, Record \& Hess (1943) registraram: placas de perfuração simples e escalariformes (em Hieronyma); raios uni e bisseriados, ocasionalmente trisseriados (em Hieronyma e Phyllanthus); raios com até 100 (ou mais) células de altura (em Alchornea, Hieronyma, Phyllanthus e Sapium); tubos lactíferos pequenos (em Sebastiania parvoniana); parênquima axial paratraqueal escasso (em Phyllanthus); fibras com pontoações de bordas conspícuas (em Hieronyma); e canais radiais frequentemente grandes, em algumas espécies de Alchornea, Croton, Euphorbia, e Sapium, entre outros gêneros.
Recentemente, Hayden \& Hayden (2000) constataram os seguintes caracteres anatômicos, em estudo de 51 gêneros, de 19 tribos de Acalyphoideae: anéis de crescimento indistintos ou fracamente delimitados; porosidade difusa; placas de perfuração simples ou com algumas escalariformes; pontoações intervasculares alternas; pontoações raio-vasculares maiores do que as intervasculares, circulares, alongadas e alternadas irregulares; parênquima difuso, difuso-em-agregados e paratraqueal-escasso, por vezes em faixas tangenciais; raios heterogêneos, raramente com mais de três células de largura; cristais prismáticos no parênquima axial e/ou raios; fibras de paredes finas a moderadamente espessas, fibrotraqueídeos não septados ou fibras libriformes. Os autores também consideraram não ser possível segregar das Acalyphoideae as subfamílias Peraceae e Pandaceae, com base em caracteres anatômicos.

Em estudo da estrutura anatômica da subfamília Euphorboideae, Mennega (2005) compara as subfamílias Acalyphoideae e Crotonoideae e discute as implicações sobre a circunscrição das Euphorbiaceae, concluindo que, apesar das três subfamílias apresentarem grande semelhança anatômica, elas podem ser segregadas pela escassa presença de laticíferos em Acalyphoideae e Crotonoideae, e pela frequente presença de laticíferos em Euphorbioideae. Afirma, ainda, que a anatomia da madeira suporta, em geral, o conceito mais restrito da família Euphorbiaceae, composta, apenas, pelas subfamílias Acalyphoideae, Crotonoideae e Euphorbioideae, excluindo Phyllanthoideae e Oldfieldioideae.

As subfamílias Acalyphoideae e Euphorbioideae, por sua vez, podem ser anatomicamente separadas pelos seguintes caracteres: vasos pequenos, numerosos, em múltiplos radiais ou em agregados; porosidade em anel, em poucos gêneros de Euphorbioideae, bem como em Necepsia e Pondadenia (Acalyphoideae); placas de perfuração simples e escalariformes, 
exclusivamente escalariformes em Microdesmis e Panda (Acalyphoideae); placas de perfuração escalariformes, ocasionais em Maprounea e em amostras jovens de Euphorbia grandiscornis (Euphorbioideae); pontoações raio-vasculares similares às intervasculares, com exceção de Euphorbia, Hura e Sapium (Euphorbiodeae), que apresentam pontoações muito largas; parênquima com distribuição semelhante, em ambas as subfamílias; e fibras septadas, praticamente ausentes em Euphorbioideae, ocasionais em Euphorbia (Mennega, 2005).

Embora muito similares às Acalyphoideae $\mathrm{e}$ Euphorbioideae, as Crotonoideae distinguemse pela presença de: vasos pequenos, geralmente médios e pouco numerosos, por vezes extremamente numerosos, com múltiplos de 2-4 poros, ou mais; porosidade difusa; placas de perfuração escalariformes, ocasionais em Codiaeum e em ramos de Givotia; pontoações intervasculares geralmente pequenas, por vezes grandes e de formato irregular em diversos gêneros; parênquima geralmente semelhante ao das Euphorboideae; raios uni e bisseriados, por vezes com até 4 células de largura, heterogêneos e com margens altas, por vezes fusionados; células perfuradas de raio, comuns; cristais e sílica, presentes em Annesijoa, Anomalocalyx, Glycydendron, Micrandra, Tetrorchidium, Cunuria e Sagotia; tubos laticíferos, presentes em Baloghia, Croton e Glycydendron; fibras não septadas, em Cnidoscolus, Jatropha e Manihot; fibras de paredes comumente finas, espessas em Leeuwenbergia, Micrandropsis e em uma espécie de Pausandra; fibras longas, na maioria dos gêneros (inferiores a $2000 \mu \mathrm{m}$ ), exceto em Leeuwenbergia $(2900 \mu \mathrm{m})$ enquanto que nas Euphorboideae o comprimento raramente excede $1200 \mu \mathrm{m}$, exceto em Elaeophorbia, Anthostema e Neoguillauminia (Mennega, 2005).

\section{MATERIAL E MÉTODOS}

No presente estudo foram anatomicamente investigadas as seguintes espécies de Euphorbiaceae lato sensu, nativas ou cultiva- das no Rio Grande do Sul: Alchornea triplinervia (Spreng.) Müll. Arg., Bernardia pulchella (Baill.) Müll. Arg., Colliguaya brasiliensis Klotzsch ex Baill., Croton dracunculoides Baill., Croton pycnocephalus Baill., Euphorbia cotinifolia L., Euphorbia pulcherrima Willd. ex Klotzsch, Euphorbia tirucalli L., Gymnanthes concolor Spreng., Hieronyma alchorneoides Allemão, Phyllanthus sellowianus (Klotzsch) Müll. Arg., Sapium glandulosum (L.) Morong., Sapium haematospermum Müll. Arg., Sebastiania brasiliensis Spreng., Sebastiania commersoniana (Baill.) L. B. Sm. \& Downs e Sebastiania schottiana (Müll. Arg.) Müll. Arg.

Para o reconhecimento de grupos distintos realizou-se uma análise de agrupamento hierárquica aglomerativa, com o auxilio do Software R pacote vegan: Community Ecology Package (Oksanen et al., 2012). Esta análise teve como base uma matriz de distância de Jaccard para dados binários, usando os caracteres anatômicos qualitativos relacionados na Tabela 1, e o método UPGMA (Unweigthed Pair-Group Method using Arithmetical Averages) como algoritmo de agrupamento (Sneath \& Sokal, 1973). Para a validação do dendrograma, calculou-se o coeficiente de correlação cofenético, o qual, segundo Valentim (2000), deve ser superior a 0,8.

Para maiores detalhes sobre a estrutura anatômica das espécies estudadas recomendamse as seguintes publicações: Denardi (2009), Marchiori et al. (2009; 2010; 2011), Siegloch et al. (2012 a,b,c) e Siegloch et al. (dados não publicados).

\section{ANÁLISE DA ESTRUTURA ANATÔMICA}

$\mathrm{Na}$ tabela 1 , vê-se que as características anatômicas de ampla ocorrência nas espécies estudadas estão de acordo com o proposto por Metcalfe \& Chalk (1972), Hayden \& Hayden (2000) e Mennega (2005): anéis de crescimento distintos; porosidade difusa; vasos predominantemente muito pequenos, solitários, em múltiplos e racemiformes, geralmente arredondados, frequentemente pouco numerosos ou mui- 
to numerosos e de comprimento médio; placas de perfuração simples; pontoações intervasculares areoladas, alternas, pequenas a grandes; pontoações raio-vasculares areoladas ou com bordas aparentemente reduzidas, por vezes radialmente estendidas ou opostas; parênquima paratraqueal-escasso, apotraqueal difuso-emagregados e, menos frequentemente, apotraqueal-escasso; raios heterogêneos, geralmente uni e bisseriados (trisseriados), compostos por células quadradas, eretas e curtamente procumbentes; células perfuradas de raios, raios fusionados e células disjuntivas, geralmente presentes; cristais e laticíferos, menos frequentes; fibras, geralmente, libriformes, delgadas e de comprimento médio, frequentemente gelatinosas.

O dendrograma (Figura 1) apresenta o agrupamento das espécies estudadas. O nível de distância com que as espécies se afastam ou se aproximam pode ser lido diretamente na coluna da esquerda, em dissimilaridade. Quanto maior o índice, menor a proximidade entre as espécies. O valor do coeficiente de correlação cofenético, de 0,86 , indica que o dendrograma é expressivo na análise.

No dendrograma (Figura 1), observa-se a divisão em dois grandes grupos: o primeiro (A), formado apenas por Hieronyma alchorneoides; e o segundo pelas demais espécies estudadas. Esse resultado já era esperado, em virtude dos estudos de Hayden \& Hayden (2000) e de Mennega (2005), favoráveis à delimitação restrita das Euphorbiaceae. Hieronyma alchorneoides, atualmente, nas Phyllanthaceae, reúne características anatômicas distintas das demais espécies analisadas: vasos longos; pontoações vasculares de difícil observação; pontoações raio-vasculares escalariformes; raios de até 4 células de largura, com células envolventes; e fibras longas, de paredes espessas.

No grupo B, representado pelas Euphorbiaceae, foram encontrados dois subgrupos, aqui designados como B1 e B2.

O subgrupo B1 compreende dois ramos: um com as espécies de Euphorbia, e outro com Alchornea triplinervia e as duas espécies de
Sapium, reunidas. Ambos se ligam a uma distância de 0,5 de dissimilaridade. Este subgrupo é formado por espécies das subfamílias Euphorboideae e Acalyphoideae. Alchornea triplinervia, única Acalyphoideae do subgrupo, mantém certa dissimilaridade com relação às espécies de Sapium. Este subgrupo se caracteriza pelos seguintes caracteres anatômicos: vasos pouco numerosos, de comprimento médio em Euphorbia e Sapium, longos em A. triplinervia; vasos de diâmetro pequeno (em $E$. cotinifolia e E. tirucalli), médio em Sapium e E. pulcherrima; pontoações intervasculares estendidas em Euphorbia e S. haematospermum, médias em Euphorbia, grandes em $A$. triplinervia e Sapium; pontoações raiovasculares estendidas em todas as espécies, opostas e com bordas reduzidas em Euphorbia e Sapium, e areoladas em A. triplinervia; parênquima apotraqueal difuso-em-agregados; raios uni e bisseridados em E. tirucalli e em Sapium, com trisseriados em A. triplinervia e E. pulcherrima, fusionados, com exceção de $A$. triplinervia.

No subgrupo B2, ocorrem três ramificações evidentes. A primeira, formada por Colliguaya brasiliensis, mantem-se a certa distância de dissimilaridade das espécies de Croton; este conjunto, pertencente às subfamílias Euphorboideae e Crotonoideae, respectivamente, apresentam os seguintes caracteres anatômicos: anéis de crescimento distintos, em $C$. brasiliensis e $C$. dracunculoides; vasos muito numerosos e curtos em $C$. brasiliensis e $C$. pycnocephalus; vasos muito pequenos, em $C$. brasiliensis e C. pycnocephalus; vasos médios, em C. dracunculoides; pontoações intervasculares areoladas, pequenas; pontoações raio-vasculares areoladas (em Croton) ou com bordas reduzidas (em C. brasiliensis); raios exclusivamente unisseriados; e presença de fibrotraqueídeos, gelatinosos em Croton. O segundo ramo é formado por Phyllanthus sellowianus (Phyllanthaceae) e Sebastiania schottiana (Euphorbiaceae-Euphorbioideae); ambas as espécies apresentam reofilia e possu- 
TABELA 1. Lista de características anatômicas para as espécies em estudo.

\begin{tabular}{|c|c|c|c|c|c|c|c|c|c|c|c|c|c|c|c|c|c|c|}
\hline CARACTERÍSTICAS & 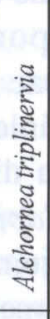 & 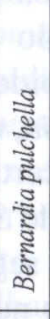 & 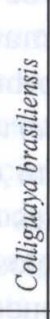 & 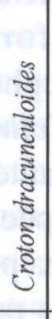 & 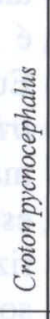 & 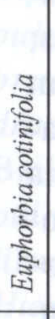 & 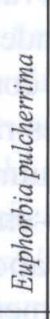 & 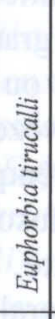 & 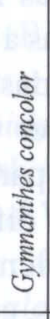 & 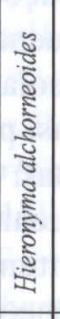 & 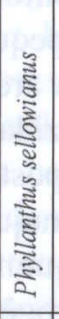 & 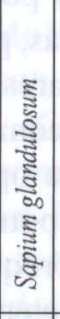 & 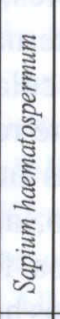 & 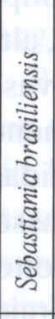 & 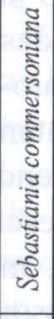 & 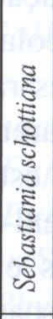 & Tot & $(\%)$ \\
\hline anéis de crescimento distintos & 1 & 0 & 1 & 1 & 0 & 1 & 1 & 1 & 1 & 1 & 1 & 1 & 1 & 1 & 1 & 1 & 14 & 87,5 \\
\hline porosidade difusa & 1 & 1 & 1 & 1 & 1 & 1 & 1 & 1 & 1 & 1 & 0 & 1 & 1 & 1 & 1 & 1 & 15 & 93,7 \\
\hline porosidade semi-difusa & 0 & 0 & 0 & 0 & 0 & 0 & 0 & 0 & 0 & 0 & 1 & 0 & 0 & 0 & 0 & 0 & 1 & 6,2 \\
\hline vasos solitários & 1 & 0 & 1 & 1 & 1 & 1 & 1 & 1 & 1 & 1 & 0 & 1 & 0 & 1 & 1 & 0 & 12 & 75 \\
\hline vasos em múltiplos radiais & 1 & 1 & 1 & 1 & 1 & 1 & 1 & 1 & 1 & 1 & 1 & 1 & 1 & 1 & 1 & 1 & 16 & 100 \\
\hline vasos racemiformes & 0 & 1 & 0 & 1 & 1 & 0 & 1 & 1 & 1 & 1 & 0 & 1 & 1 & 1 & 1 & 0 & 11 & 68,7 \\
\hline vasos com formato angular & 0 & 1 & 1 & 0 & 0 & 1 & 0 & 1 & 0 & 0 & 1 & 1 & 0 & 0 & 0 & 0 & 6 & 37,5 \\
\hline vasos com formato circular a oval & 1 & 0 & 0 & 1 & 1 & 1 & 1 & 1 & 1 & 1 & 0 & 1 & 1 & 1 & 1 & 1 & 13 & 81,2 \\
\hline $\begin{array}{l}\text { vasos pouco num erosos } \\
\left(5-20 / \mathrm{mm}^{2}\right)\end{array}$ & 1 & 0 & 0 & 0 & 0 & 1 & 1 & 1 & 0 & 1 & 0 & 1 & 1 & 0 & 0 & 0 & 7 & 43,7 \\
\hline vasos numerosos $\left(20-40 / \mathrm{mm}^{2}\right)$ & 0 & 0 & 0 & 0 & 0 & 0 & 0 & 0 & 0 & 0 & 0 & 0 & 0 & 0 & 1 & 0 & 1 & 6,2 \\
\hline $\begin{array}{l}\text { vasos muito num erosos } \\
\left(40-100 / \mathrm{mm}^{2}\right)\end{array}$ & 0 & 0 & 1 & 1 & 1 & 0 & 0 & 0 & 1 & 0 & 0 & 0 & 0 & 1 & 0 & 1 & 6 & 37,5 \\
\hline $\begin{array}{l}\text { vasos extremamente numerosos } \\
\left(\geq 100 / \mathrm{mm}^{2}\right)\end{array}$ & 0 & 1 & 0 & 0 & 0 & 0 & 0 & 0 & 0 & 0 & 1 & 0 & 0 & 0 & 0 & 0 & 2 & 12,5 \\
\hline vasos curtos $(\leq 350 \mu \mathrm{m})$ & 0 & 0 & 1 & 0 & 1 & 0 & 0 & 0 & 0 & 0 & 0 & 0 & 0 & 0 & 0 & 0 & 2 & 12,5 \\
\hline $\begin{array}{l}\text { vasos de comprimento médio } \\
(350-800 \mu \mathrm{m})\end{array}$ & 0 & 1 & 0 & 1 & 0 & 1 & 1 & 1 & 1 & 0 & 1 & 1 & 1 & 1 & 1 & 1 & 12 & 75 \\
\hline vasos longos $(\geq 800 \mu \mathrm{m})$ & 1 & 0 & 0 & 0 & 0 & 0 & 0 & 0 & 0 & 1 & 0 & 0 & 0 & 0 & 0 & 0 & 2 & 12,5 \\
\hline $\begin{array}{l}\text { vasos de diâmetro muito pequeno } \\
(\leq 50 \mu \mathrm{m})\end{array}$ & 0 & 1 & 1 & 0 & 1 & 0 & 0 & 0 & 1 & 0 & 1 & 0 & 0 & 1 & 1 & 1 & 8 & 50 \\
\hline $\begin{array}{l}\text { vasos de diâmetro pequeno } \\
(50-100 \mu \mathrm{m})\end{array}$ & 0 & 0 & 0 & 1 & 0 & 1 & 0 & 1 & 0 & 0 & 0 & 0 & 0 & 0 & 0 & 0 & 3 & 18,5 \\
\hline $\begin{array}{l}\text { vasos de diâmetro médio } \\
(100-200 \mu \mathrm{m})\end{array}$ & 1 & 0 & 0 & 0 & 0 & 0 & 1 & 0 & 0 & 1 & 0 & 1 & 1 & 0 & 0 & 0 & 5 & 31,2 \\
\hline placas de perfuração simples & 1 & 1 & 1 & 1 & 1 & 1 & 1 & 1 & 1 & 1 & 1 & 1 & 1 & 1 & 1 & 1 & 16 & 100 \\
\hline vasos com espessamentos espiralados & 0 & 1 & 1 & 0 & 0 & 0 & 0 & 0 & 0 & 0 & 0 & 0 & 0 & 0 & 0 & 0 & 2 & 12,5 \\
\hline vasos com conteúdos & 1 & 0 & 0 & 1 & 0 & 0 & 0 & 0 & 0 & 1 & 0 & 0 & 0 & 1 & 0 & 0 & 4 & 25 \\
\hline pontoações intervasculares estendidas & 0 & 0 & 0 & 0 & 0 & 1 & 1 & 1 & 0 & 0 & 0 & 0 & 1 & 0 & 0 & 0 & 4 & 25 \\
\hline pontoações intervasculares coalescentes & 0 & 0 & 0 & 1 & 0 & 0 & 1 & 1 & 0 & 0 & 0 & 0 & 0 & 0 & 0 & 0 & 3 & 18,5 \\
\hline $\begin{array}{l}\text { pontoações intervasculares alternas, } \\
\text { areoladas }\end{array}$ & 1 & 1 & 1 & 1 & 1 & 1 & 1 & 1 & 1 & 0 & 1 & 1 & 1 & 1 & 1 & 1 & 15 & 93,7 \\
\hline $\begin{array}{l}\text { pontoações intervasculares } \\
\text { pequenas }(4-7 \mu \mathrm{m})\end{array}$ & 0 & 0 & 1 & 1 & 1 & 0 & 0 & 0 & 1 & 0 & 1 & 0 & 0 & 0 & 0 & 1 & 6 & 37,5 \\
\hline $\begin{array}{l}\text { pontoações intervasculares } \\
\text { médias }(7-10 \mu \mathrm{m})\end{array}$ & 0 & 0 & 0 & 0 & 0 & 1 & 1 & 1 & 0 & 0 & 0 & 0 & 0 & 0 & 1 & 0 & 4 & 25 \\
\hline $\begin{array}{l}\text { pontoações intervasculares } \\
\text { grandes }(\geq 10 \mu \mathrm{m})\end{array}$ & 1 & 1 & 0 & 0 & 0 & 0 & 0 & 0 & 0 & 0 & 0 & 1 & 1 & 1 & 0 & 0 & 5 & 31,2 \\
\hline $\begin{array}{l}\text { pontoações intervasculares de } \\
\text { difícil observação }\end{array}$ & 0 & 0 & 0 & 0 & 0 & 0 & 0 & 0 & 0 & 1 & 0 & 0 & 0 & 0 & 0 & 0 & 1 & 6,2 \\
\hline $\begin{array}{l}\text { pontoações raio-vasculares } \\
\text { areoladas }\end{array}$ & 1 & 1 & 0 & 1 & 1 & 0 & 0 & 0 & 1 & 0 & 0 & 0 & 0 & 1 & 1 & 1 & 8 & 50 \\
\hline $\begin{array}{l}\text { pontoações raio-vasculares } \\
\text { simples ou com bordas reduzidas }\end{array}$ & 0 & 0 & 1 & 0 & 0 & 1 & 1 & 1 & 0 & 0 & 1 & 1 & 1 & 0 & 0 & 0 & 7 & 43,7 \\
\hline $\begin{array}{l}\text { pontoações raio-vasculares } \\
\text { estendidas }\end{array}$ & 1 & 1 & 0 & 0 & 0 & 1 & 1 & 1 & 0 & 1 & 0 & 1 & 1 & 0 & 0 & 0 & 8 & 50 \\
\hline $\begin{array}{l}\text { pontoações raio-vasculares } \\
\text { escalariformes }\end{array}$ & 0 & 0 & 0 & 0 & 0 & 0 & 0 & 0 & 0 & 1 & 0 & 0 & 0 & 0 & 0 & 0 & 1 & 6,2 \\
\hline $\begin{array}{l}\text { pontoações raio-vasculares } \\
\text { opostas }\end{array}$ & 0 & 0 & 0 & 0 & 0 & 1 & 1 & 1 & 0 & 0 & 0 & 1 & 1 & 0 & 0 & 0 & 5 & 31,2 \\
\hline
\end{tabular}

Caráter anatômico presente (1). Caráter anatômico ausente (0). Tot (total). \% (percentagem) 
TABELA 1. Continuação

CARACTERISTICAS

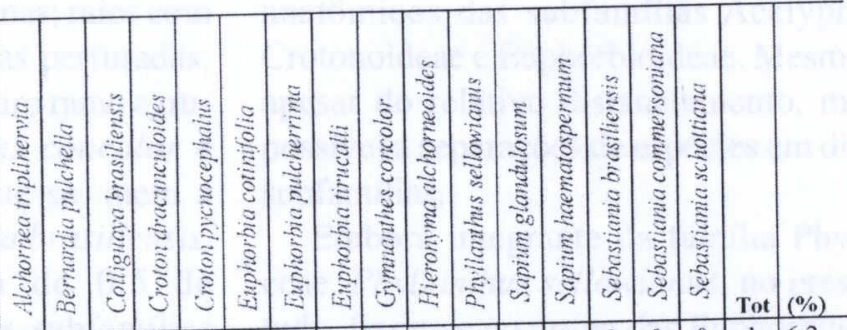

parênquima axial ausente

parênquima reticulado

parênquima apotraqueal difuso

parênquima apotraqueal

difuso-em-agregados

parênquima paratraqueal escasso

parênquima paratraqueal

vasicêntrico

parênquima com conteúdo

raios numerosos (4-12/mm)

raios muito numerosos

( $\geq 12 / \mathrm{mm})$

raios exclusivamente

unisseriados

raios uni e bisseriados

raios com 1-3 células de largura

raios com 1-4 células de largura

raios fusionados

raios agregados

raios com laticíferos

raios com células disjuntivas

raios com células perfiradas

raios com células envolventes

raios com crislais

raios heterogêneos, com células

procumbentes. quadradas e eretas

raios heterogêneos, com células

quadradas e eretas

raios com conteúdos

\begin{tabular}{lllllllllllllllllllll}
0 & 0 & 0 & 0 & 0 & 0 & 0 & 0 & 0 & 0 & 1 & 0 & 0 & 0 & 0 & 0 & 1 & 6,2 \\
\hline
\end{tabular}

raios com células mucilaginosas

fibras septadas

fibrotraqueideos

(pontoações $\geq 3$ um)

fibras libriformes

(pontoações < $3 \mu \mathrm{m}$ )

fibras curtas ( $\leq 900 \mu \mathrm{m})$

tibras médias $(900-1600 \mu \mathrm{m})$

fibras longas ( $\geq 1600 \mathrm{um}$ )

fibras com paredes delgadas

fibras com paredes finas

a espessas

fibras com paredes espessas

fibras gelatinosas

libras gelatinosas $\mathrm{em}$ laixas

\begin{tabular}{lllllllllllllllllllll}
0 & 0 & 0 & 0 & 0 & 0 & 0 & 0 & 0 & 0 & 0 & 0 & 0 & 1 & 0 & 0 & 1 & 6.2 \\
\hline
\end{tabular}

\begin{tabular}{lll|l|l|l|l|l|l|l}
0 & 0 & 1 & 0 & 0 & 1 \\
\hline & 0 & 1 & 0 & 0 & 1
\end{tabular}

$\begin{array}{llll}1 & 1 & 1 & 1\end{array}$

\begin{tabular}{lllllllll|l}
1 & 1 & 0 & 1 & 0 & 10 & 62,5
\end{tabular}

\begin{tabular}{lllllllllllllllllllllll}
1 & 1 & 1 & 1 & 1 & 1 & 0 & 1 & 1 & 1 & 0 & 1 & 1 & 0 & 0 & 0 & 11 & 68,7 \\
\hline 0 & 0 & 0 & 0 & 0 & 0 & 1 & 0 & 0 & 0 & 0 & 0 & 0 & 0 & 0 & 0 & 1 & 6,2 \\
\hline
\end{tabular}

\begin{tabular}{llllllllllllllllllllll|l}
0 & 0 & 0 & 0 & 0 & 0 & 0 & 0 & 1 & 1 & 0 & 0 & 0 & 0 & 0 & 0 & 2 & 12,5 \\
\hline 0 & 0 & 0 & 0 & 0 & 0 & 0 & 0 & 0 & 1 & 0 & 0 & 0 & 0 & 0 & 0 & 1 & 6,2 \\
\hline
\end{tabular}

\begin{tabular}{lllllllllllllllllllllllll|l}
1 & 1 & 1 & 1 & 1 & 1 & 1 & 1 & 1 & 0 & 1 & 1 & 1 & 1 & 1 & 1 & 15 & 93,7 \\
\hline
\end{tabular}

\begin{tabular}{|l|l|l|l|l|l|l|l|l|l|l|l|l|l|l|l|l|l}
0 & 0 & 1 & 1 & 1 & 0 & 0 & 0 & 0 & 0 & 0 & 0 & 0 & 0 & 0 & 0 & 3 & 18,5 \\
1 & 0 & 0 & 0 & 0 & 0 & 0 & 1 & 1 & 0 & 0 & 1 & 1 & 1 & 1 & 1 & 8 & 50 \\
\hline
\end{tabular}

(1)

\begin{tabular}{lllllllllllllllllllllllll}
1 & 0 & 0 & 0 & 0 & 0 & 0 & 1 & 1 & 0 & 0 & 1 & 1 & 1 & 1 & 1 & 8 & 50 \\
1 & 1 & 0 & 0 & 0 & 1 & 1 & 0 & 0 & 0 & 1 & 0 & 0 & 0 & 0 & 0 & 5 & 31,2 \\
\hline
\end{tabular}

\begin{tabular}{llllllllllllllllllllll}
1 & 1 & 0 & 0 & 0 & 1 & 1 & 0 & 0 & 0 & 1 & 0 & 0 & 0 & 0 & 0 & 5 & 31,2 \\
\hline 0 & 0 & 0 & 0 & 0 & 0 & 0 & 0 & 0 & 1 & 0 & 0 & 0 & 0 & 0 & 0 & 1 & 6,2 \\
\hline 0 & 1 & 0 & 0 & 0 & 1 & 1 & 1 & 1 & 1 & 0 & 1 & 1 & 0 & 1 & 0 & 9 & 56,2 \\
\hline
\end{tabular}

\begin{tabular}{llllllllllllllllllllllll}
0 & 1 & 0 & 0 & 0 & 1 & 1 & 1 & 1 & 1 & 0 & 1 & 1 & 0 & 1 & 0 & 9 & 56,2 \\
\hline 0 & 1 & 0 & 0 & 0 & 0 & 0 & 0 & 0 & 0 & 0 & 0 & 0 & 0 & 0 & 0 & 1 & 6,2 \\
\hline
\end{tabular}

\begin{tabular}{lllllllllllllllllllll}
1 & 0 & 0 & 0 & 0 & 1 & 1 & 1 & 0 & 0 & 0 & 0 & 0 & 0 & 1 & 0 & 5 & 31,2 \\
\hline
\end{tabular}

Caráter anatômico presente (1). Caráter anatômico ausente (0). Tot (total). \% (percentagem). 
em poucos caracteres anatômicos em comum: vasos de tamanho médio, muito pequenos; pontoações intervasculares pequenas; raios com células quadradas, eretas e células perfuradas; fibras curtas e gelatinosas. O terceiro ramo agrupa quatro espécies: Gymnanthes concolor e Sebastiania commersoniana, que se unem a Bernardia pulchella e Sebastiania brasiliensis, mais ou menos a distância de 0,5 de dissimilaridade; pertencentes às subfamílias Euphorbioideae e Acalyphoideae, distinguemse pelas seguintes características anatômicas: porosidade difusa; vasos de comprimento médio e diâmetro muito pequeno; pontoações intervasculares areoladas, alternas; pontoações grandes, em B. pulchella e S.brasiliensis; pontoações médias, em $S$. commersoniana; pontoações pequenas, em G. concolor; pontoações raio-vasculares areoladas; parênquima reticulado, em $S$. brasiliensis; raios heterogêneos, com células quadradas, eretas e curtamente procumbentes, em $G$. concolor e $S$. commersoniana; raios com células quadradas e eretas, em B. pulchella e S.brasiliensis; raios uni-bisseriados, com exceção de B. pulchella (trisseriados); fibras libriformes de comprimento médio, gelatinosas.

As espécies estudadas (Figura 1) não formaram grupos de acordo com suas respectivas subfamílias, corroborando estudos de Hayden
\& Hayden (2000) e de Mennega (2005), que comprovaram certa conformidade nos caracteres anatômicos das subfamílias Acalyphoideae, Crotonoideae e Euphorbioideae. Mesmo assim, apesar do relativo distanciamento, mostra-se possível a separações de espécies em diferentes subfamílias.

Embora integrante da família Phyllanthaceae, Phyllanthus sellowianus, no presente estudo, fixou-se no grupo das Euphorbiaceae, no dendrograma da Figura 1.

O dendrograma evidencia, ainda, que as espécies estudadas podem ser distinguidas entre si por caracteres anatômicos. Apesar da pequena dissimilaridade de algumas espécies, não é impossível a separação das mesmas. Euphorbia tirucalli, por exemplo, apresenta pontoações intervasculares coalescentes, raios uni e bisseriados, com células mucilaginosas e fibras de tamanho médio, separando-se, portanto, de E. cotinifolia; Croton dracunculoides, apresenta raios com cristais, pontoações intervasculares coalescentes e vasos com conteúdo, distinguindo-se, portanto, de Croton pycnocephalus; e Sapium glandulosum, apresenta fibrotraqueídeos gelatinosos, ao contrário de $S$. haematospermum. As espécies do gênero Sebatiania, no entanto, não se uniram entre si, o que demostra certa heterogeneidade dos caracteres anatômicos neste gênero.

\section{CHAVE DICOTÔMICA}

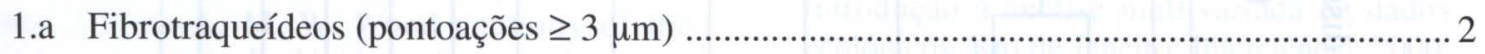

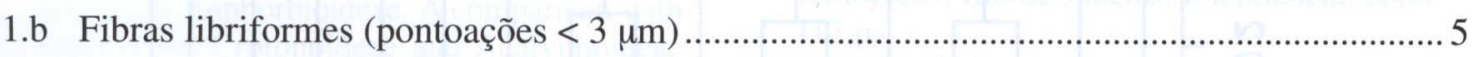

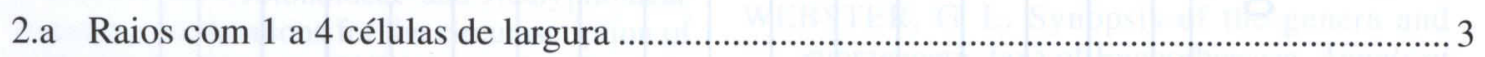

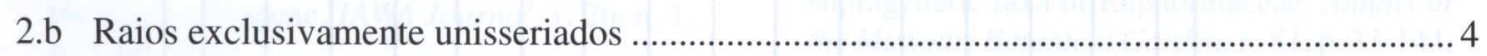

3.a Raios com até 3 células de largura ................................................... Sapium glandulosum

3.b Raios mais largos (até 4 células) ..................................................... Hieronyma alchorneoides

4.a Raios com cristais ........................................................................ Croton dracunculoides

4.b Raios sem cristais ................................................................................. Croton pycnocephalus

5.a Fibras libriformes septadas .............................................................. Phyllanthus sellowianus

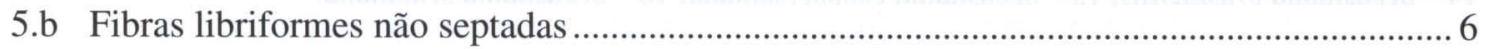


6.a Pontoações intervasculares estendidas

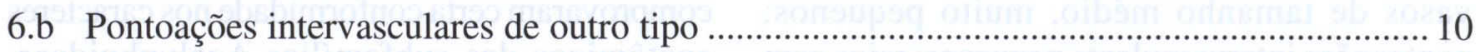

7.a Pontuações intervasculares médias

7.b Pontuações intervasculares grandes Sapium haematospermum

8.a Vasos de diâmetro pequeno 9

8.b Vasos de diâmetro médio Euphorbia pulcherrima

9.a Raios com células mucilaginosas Euphorbia tirucalli

9.b Raios sem células mucilaginosas Euphorbia cotinifolia 10.a Parênquima axial com conteúdo Gymnanthes concolor

10.b Parênquima axial sem conteúdo 11

11.a Vasos com espessamentos espiralados 12

11.b Vasos sem espessamentos espiralados

12.a Raios agregados Bernardia pulchella

12.b Raios não agregados Colliguaya brasiliensis

13.a Parênquima axial reticulado Sebastiania brasiliensis

13.b Parênquima axial apotraqueal difuso-em-agregados e paratraqueal escasso Alchornea triplinervia

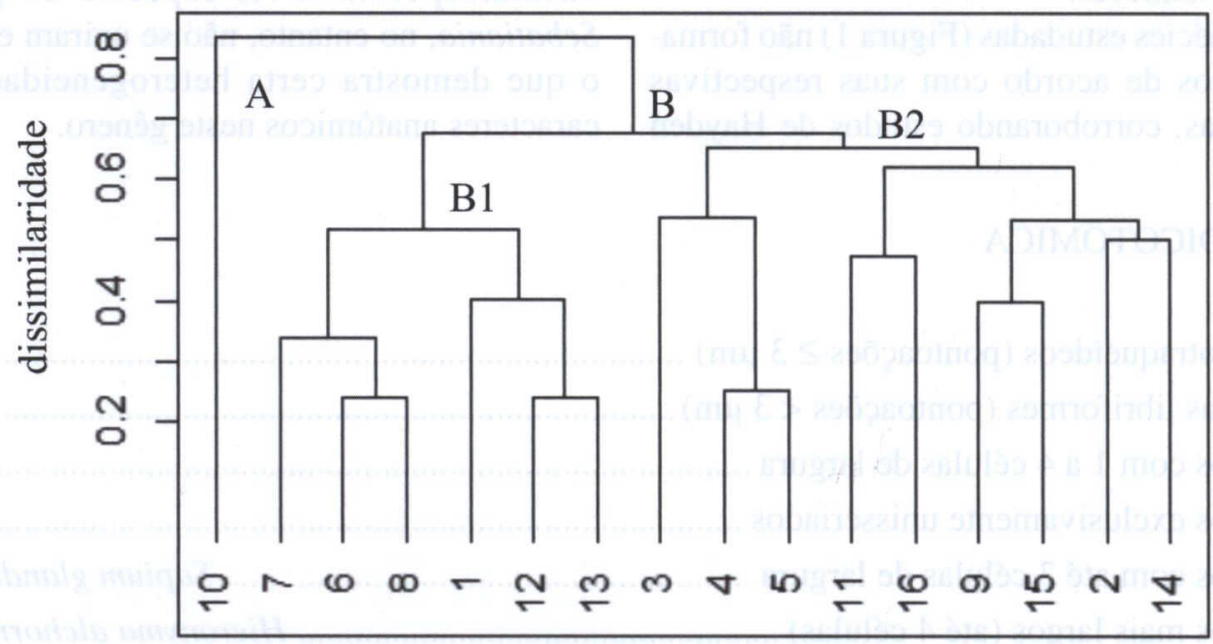

FIGURA 1. Dendrograma de agrupamento das espécies estudadas, utilizando a distância de Jaccard e o método UPGMA. 1 - Alchornea triplinervia; 2 - Bernardia pulchella; 3 -Colliguaya brasiliensis; 4 - Croton dracunculoides; 5 - Croton pycnocephalus; 6-Euphorbia cotinifolia; 7-Euphorbia pulcherrima; 8-Euphorbia tirucalli; 9-Gymnanthes concolor; 10 - Hieronyma alchorneoides; 11 - Phyllanthus sellowianus; 12 -Sapium glandulosum; 13 -Sapium haematospermum; 14 - Sebastiania brasiliensis; 15 - Sebastiania commersoniana; 16 - Sebastiania schottiana. 


\section{REFERÊNCIAS BIBLIOGRÁFICAS}

APG (The Angiosperm Phylogeny Group) II. An update of the Angiosperm Phylogeny Group classification for the orders and families of higher plants: APG II. Botanical Journal of the Linnean Society, n. 141, p. 399-496, 2003.

APG (The Angiosperm Phylogeny Group) III. An update of the Angiosperm Phylogeny Group classification for the orders and families of flowering plants: APG III. Botanical Journal of the Linnean Society, v. 161, p. 105-121, 2009.

DENARDI, L. Anatomia e flexibilidade do caule de quatro espécies lenhosas para o manejo biotécnico de cursos de água / por Luciano Denardi; orientador José Newton Cardoso Marchiori. - Santa Maria, 2007. 112 f.: il. Tese (Doutorado) - Universidade Federal de Santa Maria, Centro de Ciências Rurais, Programa de Pós - Graduação em Engenharia Florestal, RS, 2007.

HAYDEN, W. J.; HAYDEN, S. M. Wood anatomy of Acalyphoideae (Euphorbiaceae). IAWA Journal, v. 21, n. 2, p. 213-235, 2000.

JUDD, W. S.; CAMPBELL, C. S.; KELLOGG, E. A.; STEVENS, P. F.; DONOGHUE, M. J. Sistemática vegetal: um enfoque filogenético. Porto Alegre: Artmed, 2009. p. 355-359.

MARCHIORI, J. N. C.; MUÑIZ, G. I. B.; SANTOS, S. R. Madeiras do Rio Grande do Sul. 1. Descrição microscópica de 33 espécies nativas. Santa Maria: [s.n.], 2009. 80 p.

MARCHIORI, J. N. C.; MUÑIZ, G. I. B.; SANTOS, S. R. Madeiras do Rio Grande do Sul. 2. Descrição microscópica de 35 espécies nativas. Santa Maria: Anaterra, 2010.80 p.

MARCHIORI, J. N. C.; DENARDI, L.; SANTOS, S. R. Madeiras do Rio Grande do Sul. 3. Descrição microscópica de 34 espécies nativas. Santa Maria: Anaterra, 2011. 80 p.

MENNEGA, A. M. W. Wood anatomy of the subfamily Euphorbioideae. A comparison with subfamilies Crotonoideae and Acalyphoideae and the implications for the circumscription of the Euphorbiaceae. IAWA Journal, v. 26, n. 1, p. 1-68, 2005.

METCALFE, C. R.; CHALK, L. Anatomy of the Dicotyledons. Oxford: Clarendon Press, 1972. p. 404-413.

OKSANEN, J.; BLANCHET, F. G.; KINDT, R.; LEGENDRE, P.; O'HARA, R. B.; SIMPSON, G. L.; STEVENS, M. H. H.; WAGNER, H. 2012. Vegan: community ecology package.
Version 2.0-6. Available from: <http://vegan.rforge.r-project.org/ >.

R Core Team (2012). R: A language and environment for statistical computing. $R$ Foundation for Statistical Computing, Vienna, Austria. ISBN 3900051-07-0, URL http://www.Rproject.org/.

RECORD, S. J.; HESS, R. W. Timbers of the New World. New Haven: Yale University Press, 1943. $640 \mathrm{p}$.

SCHULTZ, A. R. Introdução ao estudo da Botânica Sistemática. Porto Alegre: Livraria do Globo, 1943. p. 377-383.

SIEGLOCH, A. M; MARCHIORI, J. N. C.; SANTOS, S. R. Anatomia do lenho de Bernardia pulchella (Euphorbiaceae). Balduinia. n. 34, p.28-32, 2012a.

SIEGLOCH, A. M; MARCHIORI, J. N. C.; SANTOS, S. R. Estudo anatômico do lenho de Sapium haematospermum Müll. Arg. (Euphorbiaceae). Balduinia. n.35, p. 27-31. 2012b.

SIEGLOCH, A. M; MARCHIORI, J. N. C.; SANTOS, S. R. Anatomia do lenho de duas espécies de Croton (Euphorbiaceae) do Rio Grande do Sul. Balduinia. n.38, p. 25-30, 2012c.

SNEATH, P. H. A.; SOKAL, R. R. Numerical taxonomy. San Francisco:W. H. Freeman \& Co, 1973. 573p.

SOBRAL, M.; BRACK, P.; IRGANG, B.; LAROCCA, J.; RODRIGUES, R. S. Flora arbórea e arborescente do Rio Grande do Sul, Brasil. São Carlos: RiMA: Novo Ambiente, 2006. 350 p.

SOUZA, V.C.; LORENZI, H. Botânica Sistemática: guia ilustrado para identificação das famílias de Angiospermas da flora brasileira, baseado em APG II. Nova Odessa: Instituto Plantarum, 2008. p. 358-368.

VALENTIN, J. L. Ecologia numérica: uma introdução à análise multivariada de dados ecológicos. Rio de Janeiro: Interciência, 2000. $117 \mathrm{p}$.

WEBSTER, G. L. Synopsis of the genera and suprageneric taxa of Euphorbiaceae. Annals of the Missouri Botanical Garden, n. 81, p. 33-144, 1994.

WURDACK, K. J.; HOFFMANN, P.; CHASE, M. W. Molecular phylogenetic analysis of uniovulate Euphorbiaceae (Euphorbiaceae sensu stricto) using plastid $R B C L$ and TRNL-F DNA sequences. American Journal of Botany, v. 92, n. 8, p.1397-1420, 2005. 